\title{
Engaging Mind Chemistry with Gamification: HR Practitioners Views
}

\author{
Veena Shenoy*and Doel Bhattacharya ${ }^{\dagger}$
}

\section{Abstract}

Purpose: The purpose of this study is to examine workplace gamification in the HR process. Moreover, to explore the impact of workplace gamification on employee engagement and experience.

Design/ Methodology /Approach: The current study is qualitative. The views of HR practitioners from Deloitte, TCS, Wipro, and Continental are taken through a telephonic and personal interview to understand workplace gamification in the HR process. And its impact on employee engagement and employee experience.

Findings: Workplace demographics are with changing due to the entry of tech-savvy and hyperactive millennials. Millennials get into an organization with much higher expectations in terms of work, work environment, and HR practices. Gamification is engrossed as one of the tools for employee engagement and employee experience. Gamification is a psychological imperative. Playing games keeps the brain fit, reduces stress, helps deal with cognitive overload, and also teaches work skills and team spirit.

Practical implications: The gamification made the work of HR practitioners easy. Creating user experience, involving them through practice and engagement was a challenge for HR practitioners. Now with the help of workplace gamification, feedback and rewards are more

\footnotetext{
"Assistant Professor, ISBR Business School in Bangalore, India; veena3172@gmail.com

tISBR Business School Bangalore, India; doelbhattacharya400@gmail.com
} 
transparent; it strengthens interpersonal relationships, betters employee experience, and establishes friendly competitions.

Originality/Value: The paper provides insights on gamification from HR practitioners' views. And the application of gamification in various HR-related processes such as recruitment, training, learning and development, performance management, and engagement. Hence, HR practitioners and policymakers can take a call on implementing workplace gamification in various HR processes, and that results in HR-related outcomes.

Keywords: Workplace Gamification, HR (Human Resources) Process, Employee Engagement, Employee Experience, HR (Human Resources) Practitioners.

\section{Introduction}

Engaged employees have always been productive and dedicated, and result-oriented (Andrew, 2011). Employee engagement is a challenge for Human resources practitioners today and tomorrow (Ruiz-Alba, 2019). Workplace demographics have changed in the recent past with the entry of tech-savvy and hyperactive millennials. The employee engagement rules of the past may not be suitable for the new generation workforce, and it is time to reinvent and rethink engagement strategies. Various attributes contribute to the involvement of employees; among them, gamification is considered as one of the vital tools. Gamification refers to "the process of the game-thinking and game mechanics to engage users and solve problems" (Zichermann, 2011).

There is always a connection between games and loyalty. When a person is playing, he/she is entirely engrossed and gets motivated to win incentives associated with the game. Furthermore, gamification helps in bringing all these practices in a non-game context to influence humans to behave differently. Gamification, with its innovative approach, has become an essential tool for employee engagement, retaining talent, and adding value to the motivation among employees. We should consider the gamification tool for problem-solving and motivational techniques to reach 
desired outcomes. Games are believed to enhance the user's experience, boost psychology, and bring research into the field. Gamification provides a way of transforming a monotonous job into an engaging and fun job (Garcia, 2019). Gamification tools make employees feel associated and interested in the task assigned (Raghavendran, 2015). Gamification is considered a virtual friend, motivator, and booster to achieve desired jobs. It provides insights to practitioners and policymakers to think about reinvesting employee engagement strategies' through gamification. Gamification can act as a catalyst in engaging employees. Several tools are used in an organization to incorporate the latest gamification techniques, which are mainly used to engage employees (Goffee, 2013). It is a fact that engaged employees can deliver healthy business outcomes. There is a paucity of research in gamification relating to the HR process, employee experience, and employee engagement. Hence, there is a need to explore this study connecting it with constructs proposed with a qualitative study from HR practitioners. This study contributes to the existing literature of gamification relating to constructs proposed in this study.

Gamification has led to transformation, which involves how it enhances sustainable advantage with culture and people. There is a need to shift from looking for a long-term sustainable competitive advantage to managing a portfolio with transient advantages. Gamification is essential to overcome various challenges related to high-stress levels at work, and it is immensely important to enabling a standard of trust in the organizational community. The four main pillars of workplace gamification are achievement, recognition, competition, and valuables. Significance of benefits includes supercharged innovation, value creation, faster revenue growth, higher-performing collaboration, high trust, and earned relationship capital, effective execution, and enhanced loyalty (Peters, 2018).

There are certain misconceptions regarding gamification which came up as a challenge. One of the most common misconceptions is that gamification is the same as gaming, which is not true. Gamification is the use of game elements in a non-gaming scenario to enhance engagement, motivation, and active participation. This 
methodology is used to motivate employees as well as to improve their knowledge and skills. Sometimes it is mistakenly used as a replacement for performance management, which is not the case. The introduction of unrelated games in the business process is not accountable as it will create more distractions. Gamification strategy must be accompanied by a very strong communication strategy spread across pre, post, and closure stages (Rao, 2020).

The two main areas of gamification which can serve the company better are training and mobility. In terms of training, interactive training methods that reinforce policies and culture in the form of process reminders in real-time, pop quizzes, rewards based on adherence to workplace policies. In terms of mobility, gamification offers a chance to easily track, communicate, and reward teammates no matter where they work (Newman, 2017).

Factors enhancing the motivation of employees are performance recognition, team competition, learning and developing new skills, promotion opportunities, financial incentives, and corporate values. Gamification techniques are mainly used in various spheres such as real-time performance, contact centers, sales, development teams, onboarding and development, community engagement. Customer service, learning and training, and customer app benefits include higher levels of engagement, learning, measuring employee retention and satisfaction, and also training (Kanazawa, 2019).

There are mainly two types of gamification: structural gamification and serious games. In structural gamification, gaming elements such as badges, levels, points, and leaderboard are added, whereas, in serious games, companies create a game or a simulation of a process such as training simulation. It is mainly used in recruitment, training and development, and motivation to engage users and solve problems. It allows organizations to drive continuous improvement in performance through the application of social and mobile apps that enable, enhance, and measure the impact of change in employee behavior. Combining game mechanics with technology advances and mobility helps businesses to incorporate employees aligning with corporate goals, enabling cross-collaboration, promoting competition, ensuring compliance, and allocating compensation. Gamification has helped the HR teams to a vast extent by being part of the talent and recruitment 
team, corporate culture and retention, learning and training, benefits and expense management, performance management, administrative processes, career success, wellness, and value alignment (Conscience, 2017).

Real-time examples of gamification include Google offers and its in-house currency called gooble to the best performing employees. Google also uses gamification techniques in its recruitment process as well. The company also conducts Google Code Jam Talent competitions to attract new talents. Google then offers interview calls to the best performing candidates. Zappos created an online community forum in which employees can create connections with their colleagues. Liveops is a cloud call center company in which employees can earn points based on different metrics such as the speed of completing customer calls, the total number of calls handled, and the level of customer satisfaction (Georgiou, 2020).

This study demonstrates the following research questions:

1. What is workplace gamification?

2. How is workplace gamification used in the HR process for employee engagement and employee experience?

From research questions, research objectives are developed:

1. To understand the workplace gamification from HR practitioners' views.

2. To know the relationship of workplace gamification with the HR process in creating employee engagement and employee experience.

Gamification is a rising trend in employee engagement, using a digital platform to help workers accomplish their objectives and goals. The employee plays a game that offers points, status, and rewards, which enhances their skills and goals, or the goals that the firm is trying to achieve. The premise is that we all like games, and a bit of friendly competition and the prizes that come with it. With increased social media use, mobile device popularity, and the growing sophistication of mobile technology, people stay more connected and play more games than ever before. Gamification is a good match for many workers in the world. It will make them more 
active and successful in a manner that is enjoyable, simple, and accessible. Around 55 percent of American employees claim they'd like to work for a gamification (Dupress) company.

\section{Methodology}

This study is qualitative, and in-depth interview is taken from HR practitioners from TCS, Wipro, and Deloitte to understand and examine the workplace gamification in the HR process and its impact on employee engagement and experience. The viewpoints of practitioners are mentioned below:

\subsection{Qualitative yards and Discussion}

Wipro: In the words of Mr. Mohan Kumar, Practioner head, Wipro, gamification has played an essential role in enhancing employee engagement in an organization, specifically in the form of an appraisal system. The introduction of gamification tools in the appraisal process has helped the employees and the organization. Moreover, gamification tools implemented in various stages in performance appraisal helped the HR managers and immediate superiors to decide on their appraisal quarterly and half-yearly. It has provided great epitome in talent management with implementations of various tools in the recruitment process, design thinking, and mind mapping techniques to optimize the efforts of employees in reaching organizational goals. These engagement tools adopted at Wipro enhanced the employee experience positively and reflected its benefits in the development of the HR process.

Deloitte: In the words of Deepti KS, Consultant, Deloitte, gamification has shaped up industries to a great extent. At Deloitte, the Knowit app is used to understand Deloitte's work culture and policies. Employees are given some challenges like quizzes or questionnaires based on their understanding of the Knowit app. Employees are provided with badges, awards, and points after they crack the quizzes and surveys associated with the company. It has helped in involving the employees in the process of understanding the culture at Deloitte. Various tools under the umbrella of gamification were implemented at Deloitte, such as training, learning, leadership and simulation, thinking and simulation, 
appraisal, innovation, rewards, and recognition. It has enhanced the experience of employees at Deloitte and their engagement.

TCS Nagpur, India: In the words of Revati Mulmulay, Talent Development Lead, gamification has come out to be an efficient tool for employee engagement in TCS. The transformation from traditional practices to new technology adoptions has been productive. The national qualifier test turned out to be successful, and the company considered the qualifiers for interview roles at TCS. TCS faced a lot of challenges initially when they had a manual process in place. The transformation to the tool-based process has saved the time and energy of both employees and HR managers. TCS launched Fresco Play, which is a gamified version. Each course comprises different domains such as AI, machine learning, cloud computing, and many other areas. Rewards are associated with the completion of the course, and badges, certificates, and awards are provided to employees. These rewards are considered in the appraisal process for hike and promotions. This gamified version has helped associates to explore various areas of knowledge and skill-building. This platform has also helped associates to link their learning with their careers. In the words of Manoraj Koty, HR Business Partner, TCS Hyderabad, millennials have different expectations when they hunt for jobs, and engaging them is a challenge for HR managers and practitioners. Hence TCS has implemented gamification tools in multiple processes of HR to hire employees.

The scope of development adopted by TCS, Wipro, and Deloitte are mentioned below:

In Road Traffic management, gamification can be useful to track the count of vehicles on the road and to reroute the direction of traffic. New techniques in gamification and AI helps to estimate the depth of the water level before the digging. The brainstorming technique is going on to come up with an AI machine that would take interviews. This process would give more bandwidth to HR's time and efforts. They are encouraged to use LinkedIn and social and digital platforms, which would foster more collaboration and learning. Social learning, as well as action learning project, can be brought to enhance the gamification process in which employees can write blogs sharing their practical experiences. A consistent 
feedback process with the respective manager increases the motivation factor of employees.

\section{Discussion}

There are several benefits of introducing gamification systems in an organisation. It improves team productivity and competitive spirit. These various benefits are improving productivity, increasing motivation, fostering innovation, strengthening communication processes, facilitating employee engagement, introducing innovative strategies, developing specific skills, and transmitting a corporate image. (Gamelearn, 2019) Gamification impacts employee behavior through encouragement, a sense of control, signs of action, and a sense of competition. Studies suggest that the use of gamification has made 90 percent of workers more successful. The use of game-based incentives improves participation of $48 \%$ to $72 \%$ of people who believe that gamification encourages them to work harder, and $95 \%$ of workers enjoy using gamified platforms. This feature allows for real-time feedback, objectivity, transparency, and flexibility and agility in the HR process. The steps to incorporate gamification require defining goals that a company wishes to accomplish and determination of necessary behavioral and ability changes. The game components are adapted to the workers and the workflow. Gamification can be used to educate staff, motivate staff, engage staff, optimize workflows, and attract young professionals. Excessive use of games-based encouragement can cause frustration and make workers feel overwhelmed, which are the two key risks involved in gamification. The implementation involving game elements in a work environment should be properly applied to achieve business goals (Anadea, 2018).

Learning management systems organizations have gradually embraced gamification. Neuroscientists firmly rooted their progress in results that include the positive feeling learners experience while achieving targets and receiving rewards that depend on increased dopamine secretion. The workers are inspired by this positive affirmation to accomplish more. Revisiting past accomplishments, such as won badges or a high place on the leaderboard, helps in the release of serotonin. The gameplay also 
increases endorphin levels, which decreases stress levels and induces euphoria. Gameplay activates the hippocampus, where new memories are formed, which facilitates new memory storage and recall. For a successful practice, regular constructive feedback is needed, and the material has to be delivered in various formats and types; training success has to be clearly noticeable, and competitions are excellent motivators (Intel, 2019).

\section{Implications and Conclusion}

Millennials get into organisations with much higher expectations in terms of work and work environment. They expect cognitive benefits and experience more than rational benefits, and engaging them is a challenge for HR practitioners. Hence, gamification is one of the reliable attributes in involving the mind chemistry of employees. HR practitioners can focus on the application of gamification in various HR-related processes such as recruitment, training, learning and development, performance management, engagement. They can also take a call on implementing workplace gamification across multiple HR processes, and this helps in achieving various HR-related outcomes. Gamification is a psychological imperative, and playing games keeps the brain fit, reduces stress, helps deal with cognitive overload, and also teaches work skills and team spirit. Now with the help of workplace gamification, feedback and rewards have become more transparent. This has strengthened interpersonal relationships, bettered employee experience, and created friendly competitions. Policymakers and HR practitioners should have an understanding of the multi-generation employees and their expectations; hence employee engagement strategies can be tailored as per the need of their target audience. Moreover, organisations must look into their hope to drive and sustain their engagement.

\section{References}

Anadea. (2018, January 31). How gamification in the workplace impacts employee productivity. Retrieved from Medium Start up: https:// medium.com/swlh/how-gamification-in-the-workplaceimpacts-employee-productivity-a4e8add048e6 
Andrew, O. C., \& Sofian, S. (2011). Engaging people who drive execution and organizational performance. American Journal of Economics and Business Administration, 3(3), 569.

Conscience, P. (2017, September 5). The role of gamification for the workforce.

Gamelearn. (2019). 8 benefits of gamifying your company. Retrieved from Gamelearn.

Garcia, A., Linaza, M. T., Gutierrez, A., \& Garcia, E. (2019). Gamified mobile experiences: smart technologies for tourism destinations. Tourism Review.

Georgiou, M. (2020, April 28). How workplace gamification improves employee productivity.

Goffee, R., \& Jones, G. (2013). Creating the best workplace on earth. Harvard Business Review, 91(5), 98-106.

Intel (2019, May 20). Intel Retail Edge Program. Retrieved from Intel: https:/ / retailedge.intel.com/50/blogs/2019/05/20/The-PositiveImpact-of-Gamification-on-E

Kanazawa, M. (2019). Using Gamification in Business to increase performance. mambo.IO.

Kumar, H., \& Raghavendran, S. (2015). Gamification, the finer art: fostering creativity and employee engagement. Journal of Business Strategy, 36(6), 3-12.

Newman, D. (2017). How to drive employee engagement with workplace gamification. Forbes.

Peters, R. (2018). The power of Gamification to elevate employee engagement. Mc Kinsey.

Rao, M. (2020). Gamification for employee engagement and motivation: CII Summit explores strategies for the knowledge era. YourStory. CII.

Ruiz-Alba, J. L., Soares, A., Rodríguez-Molina, M. A., \& Banoun, A. (2019). Gamification and entrepreneurial intentions. Journal of Small Business and Enterprise Development.

Saha, M. D., \& Pandita, D. (2017). Digitalizing human resources through gamification for employee engagement. ELK Asia Pacific Journals, 4.

Sarangi, S., \& Shah, S. (2015). Individuals, teams and organisations score with gamification. Human Resource Management International Digest.

Zichermann, G., \& Cunningham, C. (2011). Gamification by design: Implementing game mechanics in web and mobile apps. " O'Reilly Media, Inc.". 